\title{
Production and radiative decay of heavy neutrinos at the Booster Neutrino Beam*
}

\author{
Luis Alvarez-Ruso and Eduardo Saul Sala ${ }^{\dagger}$ \\ Departamento de Física Teórica and Instituto de Física Corpuscular \\ Centro Mixto Universidad de Valencia-CSIC, SPAIN \\ E-mail: Luis.Alvarezeific.uv.es, eduardo.sauldific.uv.es
}

\begin{abstract}
The MiniBooNE experiment reported results from the analysis of $v_{e}$ and $\bar{v}_{e}$ appearance searches, which showed an excess of signal-like events at low reconstructed neutrino energies with respect to the expected background. A proposed explanation for this anomaly assumes the existence of a heavy $(\sim 50 \mathrm{MeV})$ sterile neutrino. These $v_{h}$ would be produced by $v_{\mu}$ electromagnetic interactions with the target, through a transition magnetic dipole moment, and by neutral current interactions, due to the $v_{\mu}-v_{h}$ mixing [1,2]. A fraction of them decays radiatively inside the detector. The emitted photons are misidentified as electrons or positrons in MiniBooNE, contributing therefore to the signal.

We have studied the $v_{h}$ production by coherent and incoherent electroweak interactions on $\mathrm{CH}_{2}$ and Ar targets, present in the MiniBooNE and the Short Baseline Neutrino (SBN) detectors at Fermilab [3]. Following the $v_{h}$ propagation and subsequent decay inside the detector, we are able to obtain the energy and angular distributions of the final photons. Within the allowed range of model parameters, we have obtained the best fit to describe the MiniBooNE excess of events. To further investigate this scenario, we have calculated the expected signal at the SBN detectors. The distinctive shape and total number of photon events from this mechanism makes its experimental investigation feasible.
\end{abstract}

The European Physical Society Conference on High Energy Physics

5-12 July

Venice, Italy

${ }^{*}$ Work supported by the Spanish Ministerio de Economía y Competitividad and European Regional Development Fund under Contracts No. FIS2014-51948-C2-1-P, FIS2014-51948-C2-2-P and SEV-2014-0398, and by Generalitat Valenciana under contract PROMETEOII/2014/0068.

${ }^{\dagger}$ Speaker. 
The paradigm of three mixing neutrino flavors emerges from oscillation experiments with solar, atmospheric, reactor and accelerator neutrinos in which the square-mass differences and mixing angles have been determined with ever growing precision. Nevertheless, a number of anomalies that challenge this picture has been observed. One of them, reported by MiniBooNE, has found an excess of electron-like events over the predicted background in both $v$ and $\bar{v}$ modes [4, 5]. The excess is concentrated at $200<E_{v}^{\mathrm{QE}}<475 \mathrm{MeV}$, where $E_{v}^{\mathrm{QE}}$ is the neutrino energy reconstructed assuming a charged-current quasielastic (CCQE) nature of the events.

Existing analyses struggle to accommodate this result together with world oscillation data, even in presence of one or more families of sterile neutrinos [6]. The effect of multinucleon interactions in $E_{v}$ reconstruction is insufficient to remove the tension in global analyses [7], pointing at an explanation that does not invoke oscillations. It was suggested that an underestimated background from photons emitted in neutral current (NC) interactions could account for the excess [8]. Indeed, the MiniBooNE detector does not distinguish between electrons and single photons. However, studies considering nuclear effects and acceptance corrections $[9,10]$, obtain a number of photon-induced electron-like events which is consistent with the MiniBooNE estimate.

Gninenko proposed that additional photons could originate in the weak production of a heavy $\left(m_{h} \approx 50 \mathrm{MeV}\right)$ sterile neutrino slightly mixed with muon neutrinos, followed by its radiative decay [11] In Ref. [2] it was pointed out that the $v_{h}$ could also be electromagnetically produced, alleviating tensions in the original proposal with other data such as those from radiative muon capture measured at TRIUMF.

We have revisited the scenario presented in Ref. [2]. We compute coherent and incoherent $v_{h}$ production using present understanding of electromagnetic (EM) and weak interactions on nucleons and nuclei. For a more detailed analysis, we compare to the MiniBooNE excess of events in the originally measured electron energy and angle [12] (being the photon ones in our case) rather than in $E_{v}^{\mathrm{QE}}$. We also take into account the experimental efficiency correction available from Ref. [12].

Further insight on the nature of the MiniBooNE anomaly should be brought by the SBN program with the SBND, MicroBooNE (currently taking data) and ICARUS detectors, capable of distinguishing between electrons and photons. We have also computed the number of photon events from $v_{h}$ for the target (Argon) and geometry of the SBN detectors.

We have studied $v_{h}$ EM and weak production in the following processes

$$
\begin{gathered}
v_{\mu}, \bar{v}_{\mu}(k)+N(p) \rightarrow v_{h}, \bar{v}_{h}\left(k^{\prime}\right)+N\left(p^{\prime}\right), \\
v_{\mu}, \bar{v}_{\mu}(k)+A(p) \rightarrow v_{h}, \bar{v}_{h}\left(k^{\prime}\right)+A\left(p^{\prime}\right), \\
v_{\mu}, \bar{v}_{\mu}(k)+A(p) \rightarrow v_{h}, \bar{v}_{h}\left(k^{\prime}\right)+X\left(p^{\prime}\right) .
\end{gathered}
$$

Reaction (2) is coherent while (3) is incoherent; excited states $X$ include any number of knocked out nucleons but no meson production. The considered targets are $N=p$ and $A={ }^{12} \mathrm{C}$ for MiniBooNE $\left(\mathrm{CH}_{2}\right)$, and $A={ }^{40} \mathrm{Ar}$ for the SBN detectors.

In the EM case, following Ref. [2], we have adopted the effective interaction

$$
\mathscr{L}_{e f f}=\frac{1}{2} \mu_{t r}^{i}\left[\bar{v}_{h} \sigma_{\mu v}\left(1-\gamma_{5}\right) v_{i}+\bar{v}_{i} \sigma_{\mu v}\left(1+\gamma_{5}\right) v_{h}\right] \partial^{\mu} A^{v}
$$


in terms of a real transition coupling $\mu_{t r}^{i} ; v_{h}$ is assumed to be a Dirac fermion of mass $m_{h}$. For all the reactions under consideration, the EM amplitude can be cast as

$$
\mathscr{M}_{E M}=\frac{e \mu_{t r}^{\mu}}{2\left(q^{2}+i \varepsilon\right)} \bar{u}\left(k^{\prime}\right) q_{\alpha} \sigma^{\alpha \mu}\left(1-\gamma_{5}\right) u(k)\left\langle Y\left(p^{\prime}\right)\left|J_{\mu}^{E M}\right| N(p)\right\rangle,
$$

where $q=k-k^{\prime}=p^{\prime}-p$. EM current $\left\langle Y\left(p^{\prime}\right)\left|J_{\mu}^{E M}\right| N(p)\right\rangle$, with $Y=p, A, X$, is the same probed in the corresponding $\left(e, e^{\prime}\right)$ scattering processes. For the nucleon, it is given in terms of electric and magnetic form factors (FF), for which we have adopted standard dipole parametrizations. For coherent scattering (2), the current is proportional to the nuclear FF, obtained as the Fourier transform of the empirical charge density distribution. Finally, for the incoherent reaction we take into account particle-hole excitations in infinite nuclear matter, adapted to finite nuclei using the local density approximation.

In the weak case, the neutrino vertex has the same structure as in the Standard model, so that the amplitude

$$
\mathscr{M}_{W}=-U_{\mu h} \frac{G_{F}}{\sqrt{2}} \bar{u}\left(k^{\prime}\right) \gamma^{\mu}\left(1-\gamma_{5}\right) u(k)\left\langle Y\left(p^{\prime}\right)\left|J_{\mu}^{W}\right| N(p)\right\rangle
$$

reduces to the one for neutrino nucleus NC scattering in the limit of mixing $U_{\mu h} \rightarrow 1$ and $m_{h} \rightarrow 0$. With the weak hadronic current $\left\langle Y\left(p^{\prime}\right)\left|J_{\mu}^{W}\right| N(p)\right\rangle$ we have proceeded as with the EM current. As usual, vector FF are related to the EM ones; for the axial FF we have adopted the conventional dipole parametrization with $M_{A}=1 \mathrm{GeV}$.

Our results for the integrated cross sections (cs) on protons and ${ }^{12} \mathrm{C}$, obtained with $m_{h}=$ $50 \mathrm{MeV}, \mu_{t r}^{\mu}=2.4 \times 10^{-9} \mu_{B}$. and $\left|U_{\mu h}\right|^{2}=0.003$ [2], are given in Fig 1 . The EM cs on ${ }^{12} \mathrm{C}$ is dominated by the coherent mechanism while the incoherent one is suppressed by Pauli blocking at low $q^{2}$, where the amplitude is enhanced by the photon propagator [Eq. (5)]. On the contrary, the incoherent reaction is the largest contribution to the weak cs. Interference terms between the EM and weak amplitudes are allowed but have a negligible contribution to the cs. Similar features are observed for the ${ }^{40} \mathrm{Ar}$ target and also in the case of antineutrinos.

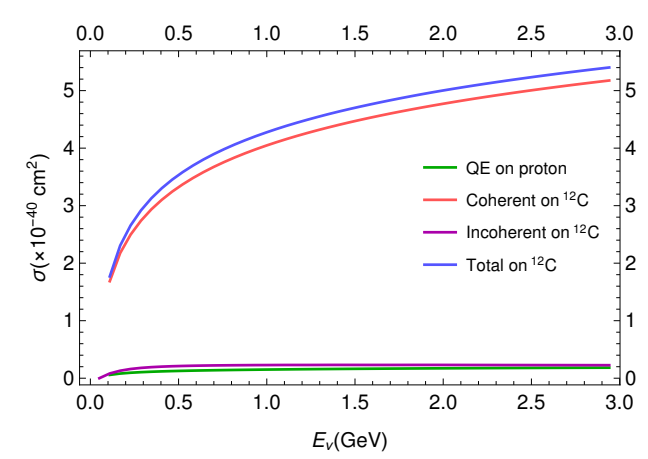

(a)

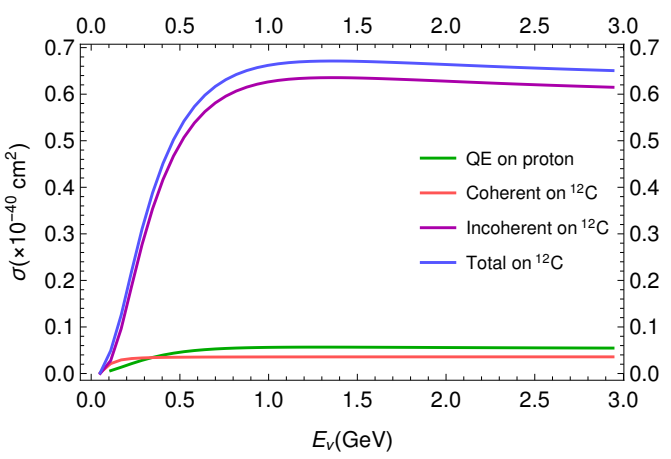

(b)

Figure 1: Integrated cross sections for $v_{h}$ production in $v_{\mu}$-nucleus scattering by EM (left) and weak (right) interactions as a function of the incident neutrino energy.

We have then investigated the $v_{h}$ propagation and radiative decay inside the detector, obtaining the photon energy and angular distributions. We have taken advantage of the fact that, as pointed 
out in Ref. [2], the beam energies are large compared to $m_{h}$ and only an insignificant amount of the electromagnetically (weakly) produced heavy neutrinos have the spin against (aligned with) its momentum. Radiative decay photons are emitted predominantly in the direction opposite to the $v_{h}$ spin. The $v_{h}$ lifetime in its rest frame $\tau=5 \times 10^{-9}$ seconds [2].

The resulting event distributions at the MiniBooNE detector for $N_{\text {POT }}=6.46 \times 10^{20}\left(N_{\text {POT }}=\right.$ $11.27 \times 10^{20}$ ) in neutrino (antineutrino) modes are shown in Fig. 2. Fluxes have been taken from

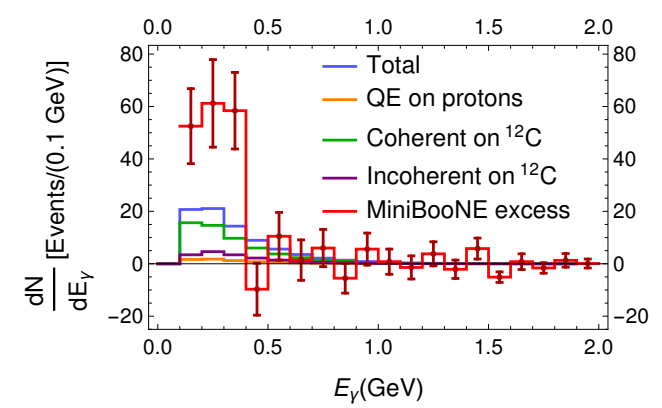

(a)

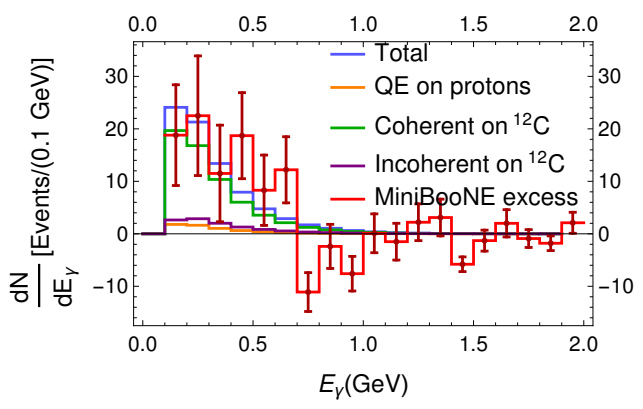

(c)

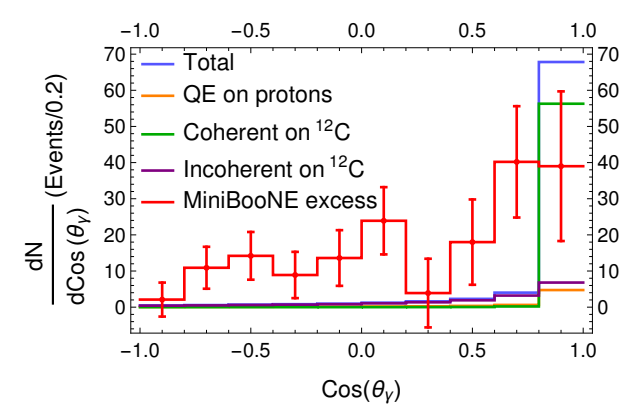

(b)

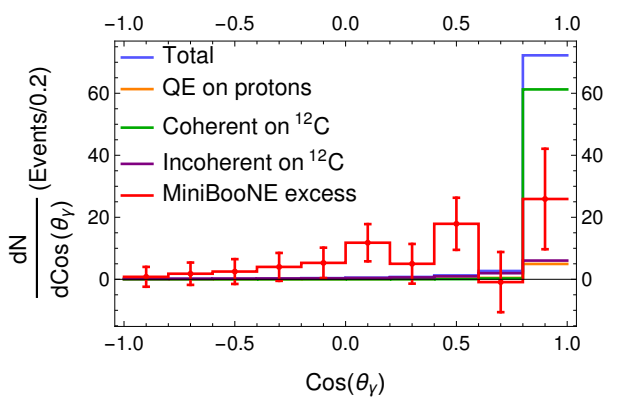

(d)

Figure 2: Photon events from radiative decay of $v_{h}, \bar{v}_{h}$ at the MiniBooNE detector in neutrino mode (top) and antineutrino mode (bottom). Theoretical results obtained with the $v_{h}$ properties of Ref. [2] are compared to the MiniBooNE excess [12].

Ref. [13]. To compare to the measured excess of events, the detection efficiency [12] has to be taken into account. Being energy dependent and low (at most $14 \%$ ), its impact on the number of events is significant. The contribution from the two protons in the $\mathrm{CH}_{2}$ target, coherent and incoherent scattering on ${ }^{12} \mathrm{C}$ are separately shown. The number of low energy events is underestimated in $v$-mode, while the agreement is good in $\bar{v}$-mode. The predominantly EM coherent contribution is strongly forward peaked. This leads to a very narrow angular distribution not observed in the experiment. This result is in line with the findings of Ref. [14].

The agreement can be improved by fitting the parameters in the allowed range established in Ref. [1]. With these values, $m_{h}=68.6 \mathrm{MeV}, \mu_{t r}^{\mu}=6.4 \times 10^{-10} \mu_{B} .,\left|U_{\mu h}\right|^{2}=0.01$ and $\tau=$ $2.5 \times 10^{-9}$ seconds, the resulting event energy and angular distributions are given in Fig. 3 . The MiniBooNE excess of events is now better described, particularly the angular distributions. This better agreement is obtained at the price of reducing the EM strength, while increasing the NC one by setting $\left|U_{\mu h}\right|$ to its maximal allowed value; this upper limit in $\left|U_{\mu h}\right|$ prevents from obtaining a more satisfactory description of the data. 


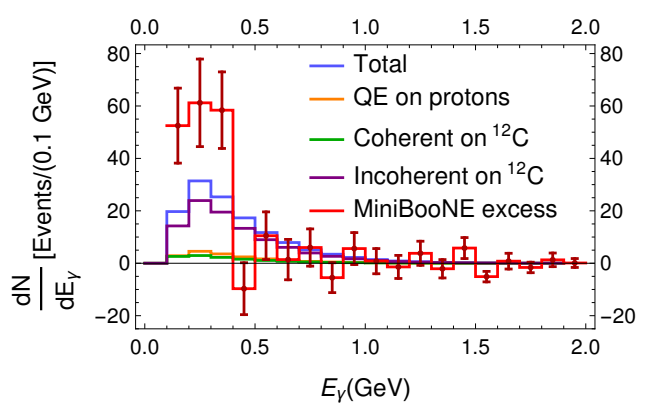

(a)

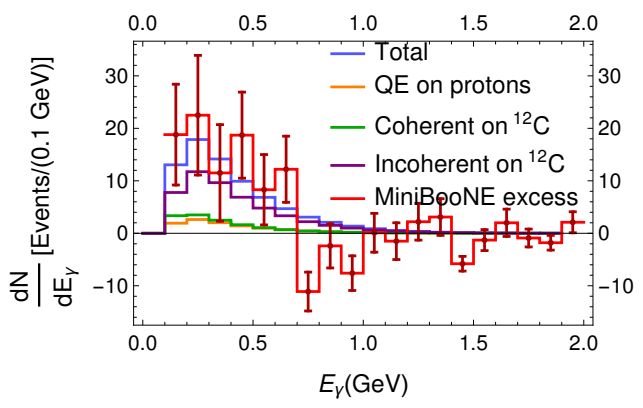

(c)

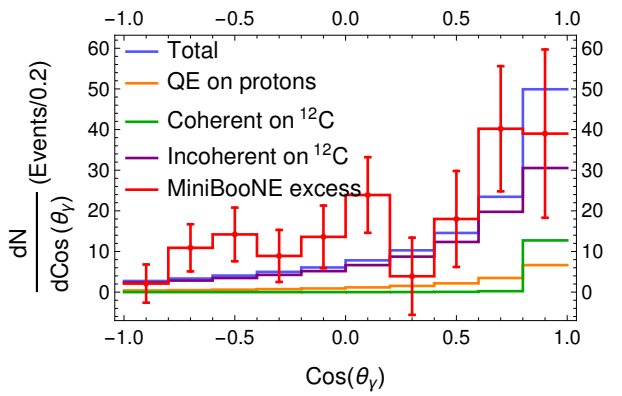

(b)

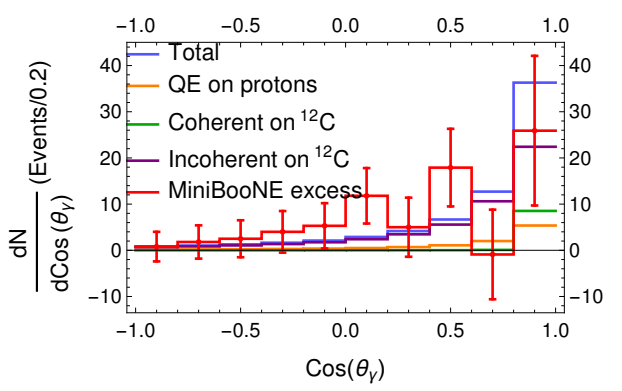

(d)

Figure 3: Photon events from radiative decay of $v_{h}, \bar{v}_{h}$ at the MiniBooNE detector in neutrino mode (top) and antineutrino mode (bottom). Theoretical results obtained with a best fit in the allowed parameter region are compared to the MiniBooNE excess [12].

The radiative decay hypothesis can be further tested at the SBN detectors. Our predictions for the photon distributions at MicroBooNE, with the flux in $v$-mode [15] and assuming $N_{\text {РОT }}=6.6 \times$ $10^{20}$, are displayed in Fig. 4. Similar results for the SBND and ICARUS detector will be reported elsewhere [3]. The shape and number of events appears distinctive from those of conventional photon emission mechanisms.

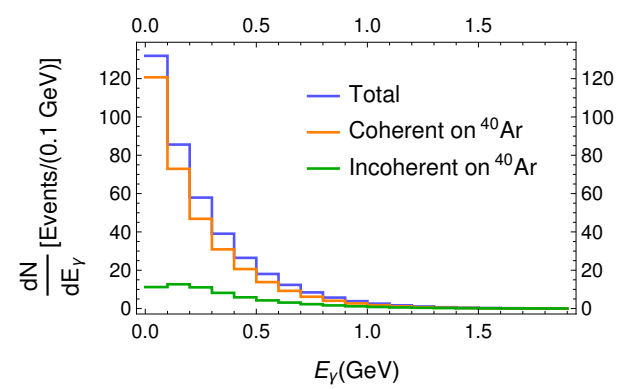

(a)

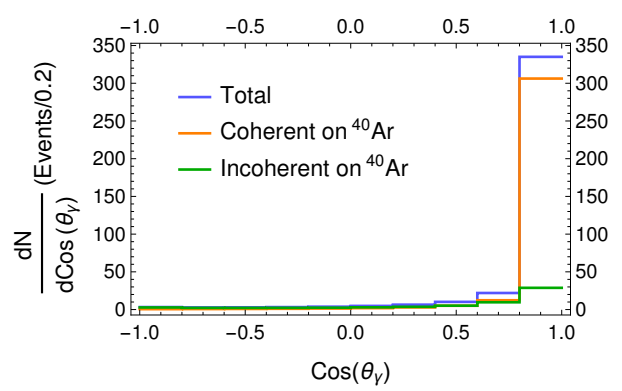

(b)

Figure 4: Photon events from radiative decay of $v_{h}$ at MicroBooNE in neutrino mode predicted with the $v_{h}$ properties of Ref. [2]. 


\section{References}

[1] S. N. Gninenko, A resolution of puzzles from the LSND, KARMEN, and MiniBooNE experiments, Phys. Rev. D83 (2011) 015015, [1009.5536].

[2] M. Masip, P. Masjuan and D. Meloni, Heavy neutrino decays at MiniBooNE, JHEP 1301 (2013) 106.

[3] L. Alvarez-Ruso and E. Saul-Sala, "in preparation.”.

[4] MiniBoonE collaboration, A. A. Aguilar-Arevalo et al., Unexplained Excess of Electron-Like Events From a 1-GeV Neutrino Beam, Phys. Rev. Lett. 102 (2009) 101802.

[5] MiniBooNE collaboration, A. Aguilar-Arevalo et al., Improved Search for $\bar{v}_{\mu} \rightarrow \bar{v}_{e}$ Oscillations in the MiniBooNE Experiment, Phys.Rev.Lett. 110 (2013) 161801.

[6] C. Giunti, M. Laveder, Y. F. Li and H. W. Long, Pragmatic View of Short-Baseline Neutrino Oscillations, Phys. Rev. D88 (2013) 073008.

[7] M. Ericson, M. V. Garzelli, C. Giunti and M. Martini, Assessing the role of nuclear effects in the interpretation of the MiniBooNE low-energy anomaly, Phys. Rev. D93 (2016) 073008.

[8] R. J. Hill, On the single photon background to $v_{e}$ appearance at MiniBooNE, Phys. Rev. D84 (2011) 017501.

[9] X. Zhang and B. D. Serot, Can neutrino-induced photon production explain the low energy excess in MiniBooNE?, Phys. Lett. B719 (2013) 409-414, [1210 . 3610].

[10] E. Wang, L. Alvarez-Ruso and J. Nieves, Single photon events from neutral current interactions at MiniBooNE, Phys.Lett. B740 (2015) 16-22.

[11] S. N. Gninenko, The MiniBooNE anomaly and heavy neutrino decay, Phys. Rev. Lett. 103 (2009) 241802.

[12] MiniBooNE collaboration:

https://www.boone.fnal.gov/for_physicists/data_release/.

[13] MiniBoonE collaboration, A. Aguilar-Arevalo et al., The Neutrino Flux prediction at MiniBooNE, Phys.Rev. D79 (2009) 072002.

[14] A. Radionov, Constraints on electromagnetic properties of sterile neutrinos from MiniBooNE results, Phys. Rev. D88 (2013) 015016.

[15] Z. Pavlovic and O. Palamara, "private communication.”. 